\title{
Load capacity of a new rope-climbing robot
}

\author{
Hao Yin ${ }^{1,2, *}$, Peiqing $\mathrm{Ye}^{1}$, Tong Zhao ${ }^{1}$ and Hui Zhang ${ }^{1}$ \\ ${ }^{1}$ School of Mechanical Engineering, Tsinghua University, Beijing 100084, China \\ ${ }^{2}$ Qingdao Campus, Naval Aeronautical University, Qingdao 266041, China
}

\begin{abstract}
Theoretical analysis and experimental research are carried out on the load capacity of one new kind rope-climbing robot, this robot could lift load along the rope depends on the friction between rope and wheel of the robot. The classic Euler formula is based on the assumption of constant friction coefficient, and this paper establishes a new load capacity model based on nonconstant friction coefficient model, presents a method to measure the distribution of friction coefficient, and builds the experimental platform to conduct experimental research on the friction coefficient and load capacity. Experimental results show that the friction coefficient decreases with the increase of wrap angle; the new model fits the results better than Euler formula.
\end{abstract}

\section{Introduction}

Friction transmission is widely used in civil and industrial fields, mine hoist, elevator and belt drive machine are some common typical forms of friction transmission ${ }^{[1-3]}$, the rope-clibing robot to be studied in this paper is another application of friction transmission. The ropeclimbing robot transports loads along the rope relied on the friction between its wheel and rope, and load capacity is a very important measure of the performance of the robot. For this kind rope-climbing robot, its load capacity equals to the maximum friction between rope and wheel, Euler formula is widely used to calculate the friction between wheel and rope or blet or other forms, but there is some deviation between the actual force and calculated values, especially for the rope-climbing robot. Most studies had applied Euler formula to different areas ${ }^{[1-5]}$, some studies had modified Euler formulation for their special materials and structures ${ }^{[6,7]}$, but it's assumed that the friction coefficient between rope and wheel keeps constant in these studies, the assumption is too simplified to correspond to reality. The rope-climbing robot as the research object, the load capacity of the robot and the distribution rules of friction coefficient between rope and wheel will be studied in this paper.

\section{Theoretical analysis of load capacity}

\subsection{Theoretical analysis based on constant friction coefficient}

Analyze the force of the rope wound on the wheel, mark the wrap angle $\varphi$, the radius of rope $r$ and the wheel's $R$, wheel angular velocity $\omega$, force at the end-A $F_{A}$ and end-B $F_{B}\left(F_{A}>F_{B}\right)$. Select a micro-segment of rope (radian $\mathrm{d} \alpha$ ) at point-D where arc-distance $\alpha$ from end-B,

\footnotetext{
* Corresponding author: yin-h14@mails.tsinghua.edu.cn
}

Fig. 1 shows the forces acting on this micro-segment, these forces can be expressed by the following equation.

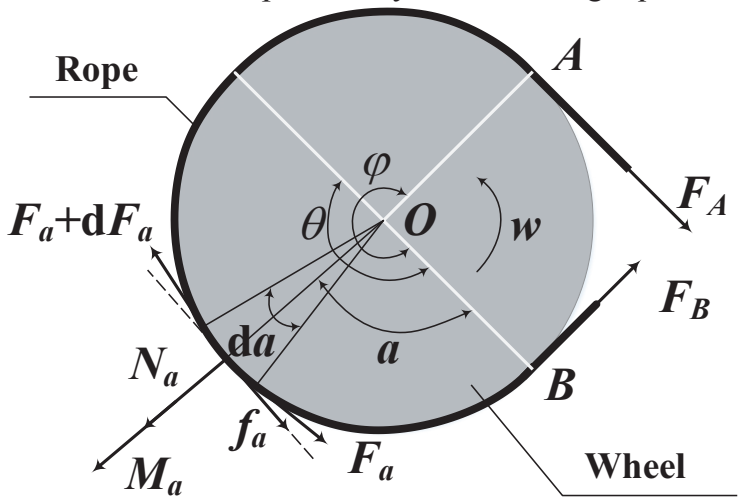

Figure 1. Working principle schematic diagram.

$$
\left.\begin{array}{l}
\left(F_{\alpha}+\mathrm{d} F_{\alpha}\right) \cos \frac{\mathrm{d} \alpha}{2}=F_{\alpha} \cos \frac{\mathrm{d} \alpha}{2}+f_{\alpha} \\
\left(2 F_{\alpha}+\mathrm{d} F_{\alpha}\right) \sin \frac{\mathrm{d} \alpha}{2}=N_{\alpha}+M_{\alpha} \\
M_{\alpha}=\rho \cdot \omega^{2} \cdot r^{2} \cdot \mathrm{d} \alpha \\
f_{\alpha}=\mu_{\alpha} \cdot N_{\alpha}
\end{array}\right\}
$$

In the equation, $f_{\alpha}, M_{\alpha}, N_{\alpha}$ are the friction, centrifugal force and support force acting on the microsegment rope, $\mu_{\alpha}$ is the friction coefficient between the rope and wheel surface at this position and moment, $\rho$ is the quality of the rope per unit length. Suppose that the friction coefficient is constant over the entire wrap angle ${ }^{[8]}$, which could be described by formula $\mu_{\alpha}=\mu \quad, \quad \alpha \in[0, \varphi] \quad, \quad$ owing to $\mathrm{d} \alpha \approx 0$, $\cos \frac{\mathrm{d} \alpha}{2}=1, \sin \frac{\mathrm{d} \alpha}{2}=\frac{1}{2} \mathrm{~d} \alpha$, ignore the second order small quantity $\mathrm{d} F_{\alpha} \sin \frac{\mathrm{d} \alpha}{2}$, 


$$
\frac{F_{A}-\rho \omega^{2} r^{2}}{F_{B}-\rho \omega^{2} r^{2}}=\mathrm{e}^{\mu \varphi}
$$

Because $\rho$ and $\omega$ are small, so $M_{\alpha}$ can be ignored,

$$
\frac{F_{A}}{F_{B}}=\mathrm{e}^{\mu \varphi}
$$

Eq.(3) is called Euler's formula because it was deduced by Euler firstly, as the classical formula for calculating the belt (or rope) - wheel friction, it is widely used in mine hoist, elevators, automotive belt transmission and so on. However, the mechanical properties of the rope used for the climbing robot is special, Coulomb friction hypothesis does not hold in the global context, so it is necessary to analyze the problem under the premise of non-constant friction coefficient .

\subsection{Theoretical analysis based on non-constant friction coefficient}

Although the friction coefficient is not constant in the global context, but focus on the micro-segment rope at the very moment, the local friciton coefficient could be seen as a constant and the relationship between the forces at both ends of the micro-segment could be described accurately by Euler Formula. Divide evenly the entire wrap angle to $\mathrm{n}$ copies, mark the friction coefficient in number $i$ wrap angle $\mu_{i-1, i}$ and the average friction coefficient $\mu_{i, j}$ in wrap angle $\varphi_{i, j}$, they should satisfy the following equations:

$$
\begin{aligned}
F_{1}= & F_{0} \mathrm{e}^{\mu_{0,1} \varphi_{0,1}} \\
& \vdots \\
F_{i}= & F_{i-1} \mathrm{e}^{\mu_{i-1, i} \varphi_{i-1, i}}=F_{0} \mathrm{e}^{\sum_{m=1}^{i} \mu_{m-1, m} \varphi_{m-1, m}}=F_{0} \mathrm{e}^{\mu_{0, i} \varphi_{0, i}} \\
& \vdots \\
F_{k}= & F_{k-1} \mathrm{e}^{\mu_{k-1, k} \varphi_{k-1, k}}=F_{0} \mathrm{e}^{\sum_{m=1}^{k} \mu_{m-1, m} \varphi_{m-1, m}}=F_{0} \mathrm{e}^{\mu_{0, k} \varphi_{0, k}} \\
& \vdots \\
F_{j}= & F_{j-1} \mathrm{e}^{\mu_{j-1, j} \varphi_{j-1, j}}=F_{0} \mathrm{e}^{\sum_{m=1}^{j} \mu_{m-1, m} \varphi_{m-1, m}}=F_{0} \mathrm{e}^{\mu_{0, j} \varphi_{0, j}} \\
& \vdots \\
F_{n}= & F_{0} \mathrm{e}^{\mu_{0, n} \varphi_{0, n}}
\end{aligned}
$$

From Eq.(4), the friction coefficients satisfy the following equations:

$$
\begin{aligned}
\mu_{0, i} \varphi_{0, i} & =\sum_{m=1}^{i} \mu_{m-1, m} \varphi_{m-1, m} \\
\mu_{0, i} & =\frac{1}{i} \sum_{m=1}^{i} \mu_{m-1, m}
\end{aligned}
$$

As Eq.(6) shows, the average friction coefficient of a wrap angle from beginning numerically equals to the equal weighted average of friction coefficients of all micro-segments rope, and it is applicable for the average friction coefficient of any wrap angle anywhere. Expand the equation $F_{j}$ :

$$
\begin{gathered}
F_{j}=F_{j-1} \mathrm{e}^{\mu_{j-1, j} \varphi_{j-1, j}}=F_{j-2} \mathrm{e}^{\mu_{j-1, j} \varphi_{j-1, j}+\mu_{j-2, j-1} \varphi_{j-2, j-1}}=\cdots \\
=F_{i} \mathrm{e}^{\sum_{m=i+1}^{j} \mu_{m-1, m} \varphi_{m-1, m}}=F_{i} \mathrm{e}^{\mu_{i, j} \varphi_{i, j}} \\
\mu_{i, j} \varphi_{i, j}=\sum_{m=i+1}^{j} \mu_{m-1, m} \varphi_{m-1, m}=\ln F_{j}-\ln F_{i} \\
\mu_{i, j}=\frac{1}{\varphi_{i, j}} \sum_{m=i+1}^{j} \mu_{m-1, m} \varphi_{m-1, m}=\frac{1}{j-i} \sum_{m=i+1}^{j} \mu_{m-1, m}
\end{gathered}
$$

Similarly:

$$
F_{j}=F_{k} \mathrm{e}^{\mu_{k, j} \varphi_{k, j}}=F_{k} \mathrm{e}^{\sum_{m=k+1}^{j} \mu_{m-1, m} \varphi_{m-1, m}}
$$

For any $F_{j}$, it can be seen obtained from initial force $F_{i}$ after wrap angle $\varphi_{i, j}(i<j)$, or from initial force $F_{k}$ after wrap angle $\varphi_{k, j}(k<j)$, or from any initial force after corresponding wrap angle, in other words, the effect of wrap angle and initial force are equivalent, initial force can be seen as a special case of wrap angle, thus discussion about the effect of wrap angle is sufficient. Denote the distribution function of friction coefficient along the wrap angle as $\mu(\varphi)$ :

$$
F_{\varphi}=F_{0} \mathrm{e}^{\int^{\varphi} \mu(\varphi) \mathrm{d} \varphi}
$$

Or:

$$
\int_{0}^{\varphi} \mu(\varphi) \mathrm{d} \varphi=\ln F_{\varphi}-\ln F_{0}
$$

Let $F_{0}=1 \mathrm{~N}$, so $\ln F_{0}=0$, thus Eq.(12) turns to:

$$
\int_{0}^{\varphi} \mu(\varphi) \mathrm{d} \varphi=\ln F_{\varphi}
$$

Or:

$$
\frac{\mathrm{d} \ln F_{\varphi}}{\mathrm{d} \varphi}=\mu(\varphi)
$$

Eq.(11) could be used to calculate the load capacity under the condition of non-constant friction coefficient, and Eq.(14) is the theoretical inference of the friction coefficient distribution.

\section{Experimental Research}

The theoretical derivation is given above; measure the static friction of different wrap angles in experiments, the function of friction coefficient along wrap angle could be obtained by computing, fitting and deriving the data. In actual use, the end tension (initial force) would be larger than $1 \mathrm{~N}$, and its curve is part of the complete curve.

Fig.2 shows the experiment platform, in the experiments, end tension $F_{B}$ would be applied by weights hung at one end of the rope, the signal of $F_{A}$ is received by mechanical sensor fixed at the other end, and then transferred by a transmitter to PC. 


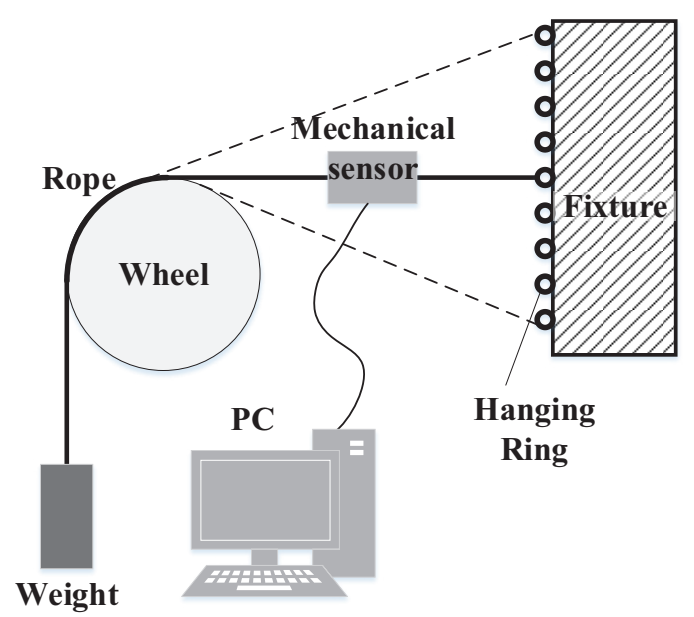

Figure 2. Experimental schematic diagram.

The experimental procedure is as follows:

1. Start the motor a few seconds after the weights hung stably;

2. Increase the motor's speed gradually until the rope slips globally in the wheel surface;

3. Shut down the motor.

Record the corresponding $F_{A}$ signal of whole process above. Use a new rope segment for each test to avoid the effects of wear on the test results, repeat the test at least 15 times under the same conditions, the average $F_{A}$ obtained from 15 values is seen as the load capacity under this condition. Change the wrap angle by adjusting the position of the sensor along the hanging ring rail on the fixture, and measure load capacity under new conditions.

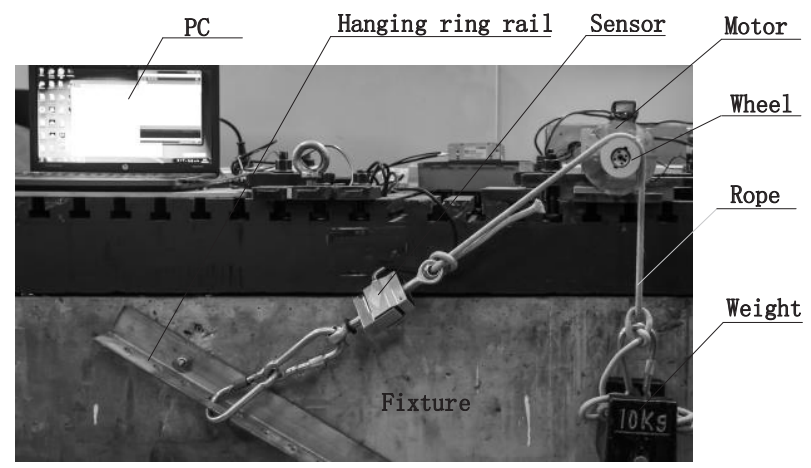

Figure 3. Experiment platform photo.

Draw the curve of load capacity changed with wrap angle, the results are shown in Fig.4. It is obvious that the load capacity and the wrap angle are approximately exponentially related, but the error will become larger as the wrap angle increases; the average coefficient of static friction decreases with the wrap angle increasing.

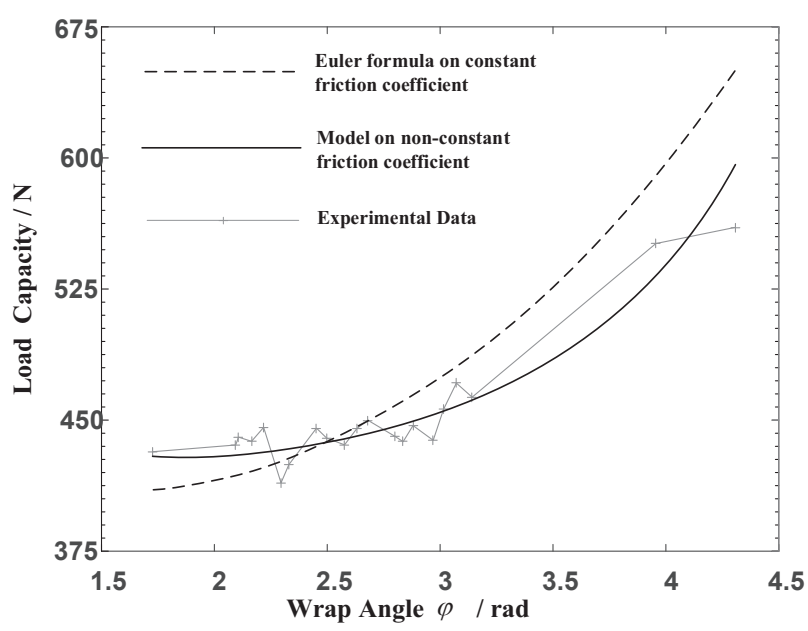

Figure 4. Load capacity trend with wrap angle.

It can be inferred that the function $\mu(\varphi)$ decreases monotonically with the wrap angle and tends to a constant value, that is, its first derivative should be negative and gradually approach 0 ; and its integral function should increase monotonically according to its physical meaning, construct a $\mu(\varphi)$ function of the following form:

$$
\mu(\varphi)=a \mathrm{e}^{b \varphi}+c
$$

In the above formula, there is $a>0, b<0, c>0$. Select the appropriate parameter, the error between model predictive value and actual value is very small, it indicates that when the wrap angle increases to a certain extent, the effect of wrap angle on friction is very slight, $\ln F_{A}$ increases linearly with the wrap angle.

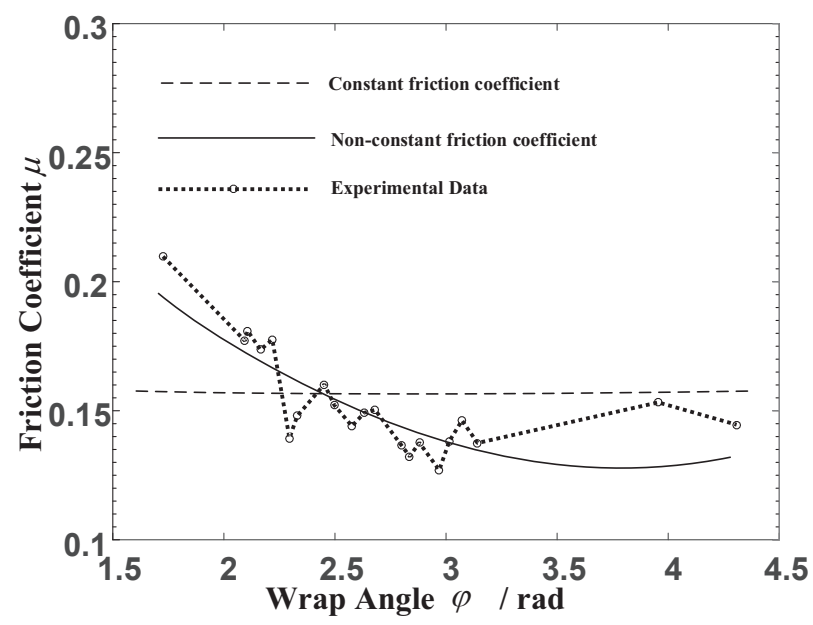

Figure 5. Friction coefficient trend with wrap angle.

\section{New model of load capacity}

According to Eq.(13) and Eq.(14), the curve in Fig.5 is just a part of the complete curve starts from point $(0,1)$. On the complete curve, the load force corresponds to the wrap angle, as shown in Fig.6, a force $F_{B}$ corresponds to angle $\varphi_{B}$, and the warp angle is $\varphi$, the start point of the integral is $B\left(\varphi_{B}, \mu_{B}\right)$, and the end point is $A\left(\varphi_{A}, \mu_{A}\right)$, where $\varphi_{A}$ and $\varphi_{B}$ satisfy $\varphi_{A}=\varphi_{B}+\varphi$. 


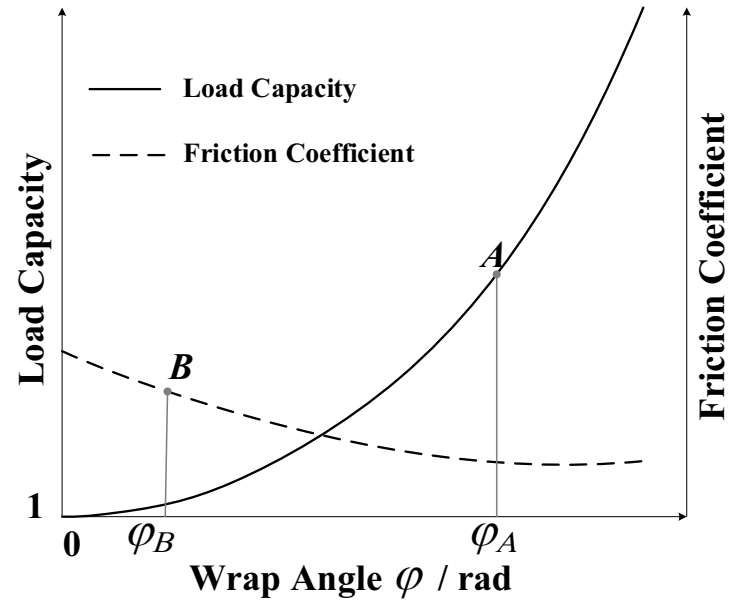

Figure 6. The complete curve.

Eq.(15) show the new model of the distribution of friction coefficient along the wrap angle, and the force can be calculated by Eq.(16), the two formula together form the load capacity mathematical model.

$$
F_{A}=F_{B} \mathrm{e}^{\int_{\varphi_{B}}^{\varphi_{A}} \mu(\varphi) \mathrm{d} \varphi}
$$

\section{Conclusion}

Theoretical derivation and experimental analysis are carried out on the load capacity of a new kind of ropeclimbing robot, mathematic model of the load capacity is obtained considering non-constant friction coefficient, and a measuring method of the friction coefficient distribution is proposed. The experimental results show the friction coefficient decreases with the increase of wrap angle; and the new model has higher precision than Euler formula.
This work was financially supported by the Foundation of The State Key Laboratory of Tribology, Tsinghua University. Project No. SKLT09B08.

\section{References}

1. Berna Balta, Fazil O. Sonmez, Abdulkadir Cengiz. Speed losses in V-ribbed belt drives[J]. Mechanism and Machine Theory, 2015, 86: 1-14.

2. Nilabh Srivastava, Imtiaz Haque, Transient dynamics of metal V-belt CVT: Effects of band pack slip and friction characteristic[J]. Mechanism and Machine Theory, 2008, 43(4): 459-479.

3. Gang Sheng, Jonah H. Lee, Vikram Narravula, et al. Experimental characterization and analysis of wet belt friction and the vibro-acoustic behavior[J]. Tribology International, 2011,44(3): 258-265.

4. Davies P, François M, Lacotte N, et al. An empirical model to predict the lifetime of braided HMPE handling ropes under cyclic bend over sheave (CBOS) loading[J]. Ocean Engineering, 2015, 97: 74-81.

5. Kastratović G, Vidanović N, Bakić V, et al. On finite element analysis of sling wire rope subjected to axial loading[J]. Ocean Engineering, 2014, 88: 480-487.

6. Weller S D, Davies P, Vickers A W, et al. Synthetic rope responses in the context of load history: Operational performance[J]. Ocean Engineering, 2014, 83: 111-124.

7. Leech $\mathrm{C}$ M. The modelling of friction in polymer fibre ropes[J]. International Journal of Mechanical Sciences 44 (2002) 621-643.

8. Valentin L. Popov. Contact Mechanics and Friction Physical Principles and Applications[M]. Tsinghua University, Firsted. Beijing, 2011.

\section{Acknowledgments}

